\section{Distinctive feature control of decision time: Same-different judgments of simultaneously heard phonemes}

\author{
RONALD A. COLE and BRIAN SCOTT \\ University of Waterloo, Waterloo, Ontario, Canada
}

Ss decided whether dichotically presented consonant or vowel phonemes were "same" or "different" in a reaction time (RT) task. Results indicated that "different" responses were made on the basis of a serial, self-terminating scan of distinctive feature differences between phonemes. "Same" responses were considered too fast to be accounted for by this process and were discussed as a separate parallel process. Recognition of dichotically presented syllables appears to involve a third process in which phonemes are also identified in terms of their distinctive features.
It is a general rule that the more different two stimuli are, the faster one may decide that they are different (Egeth, 1966; Bamber, 1969). Experiments that have tested this notion by examining reaction time (RT) to successively heard phonemes differing by few or many distinctive features have yielded ambiguous results. For example, McInish and Tikofsky (1969) presented Ss with successive pairs of spoken consonants (paired with $/ a /$ ) that either were the same or differed by one or two distinctive features, according to the Miller-Nicely (1955) distinctive feature system. Ss were required to decide, as quickly as possible, whether the second consonant was the same as the first. It was found that RT was fastest for "same" responses, and faster for pairs of consonants that differed by two, rather than one, distinctive features.

In a similar procedure, Cole (unpublished data) presented Ss with pairs of spoken consonants (C, D, P, $\mathrm{T})$ or vowels $(\mathrm{E}, \mathrm{A}, \mathrm{O}$, "OO") separated by $.5,2$, or 8 sec in a same-different RT task. While "same" responses were faster than "different" responses, no differences were found in RT to consonants or vowels differing by one, two, or three distinctive features.

It is possible that Ss in Cole's experiment originally identified each spoken letter in terms of its distinctive features, but recoded each syllable into a higher-order response. This could be accomplished, for example, if $S$ generated the letter name corresponding to each syllable (e.g., "p" for /pi//) prior to the presentation of the second syllable. In this case, when $\mathbf{S}$ was presented with the second syllable, he would convert this stimulus to a letter in order to make a same-different judgment. Therefore, one would not expect RT to vary as a function of the distinctive-feature similarity of the two syllables.

There is some evidence to support this notion. Chananie and Tikofsky (1968) found no difference in the RT to successively heard single-syllable words which differed in their initial consonants by one, two, three, or four distinctive features. This result suggests that phonemes were recorded into word representatives in auditory memory and these word representatives could be compared without reference to individual phonemes or their distinctive features.

One way to decrease the possibility that $S$ s use the time between successive presentations of a stimulus to recode a phoneme from a set of distinctive features to a name code is to present two phonemes simultaneously during a recognition task. The present experiment was designed to test this hypothesis for dichotically presented pairs of consonants and vowels. It was predicted that the more different two consonants or vowels are in terms of their distinctive-feature composition, the faster $\mathbf{S}$ will decide that they are different.

\section{EXPERIMENT 1}

\section{Method}

Subjects. Forty right-handed undergraduate students from the University of Waterloo served as Ss. Their participation partially fulfilled a course requirement in introductory psychology.

Stimuli. The consonant phonemes /b, $m, v, g, d, n, z, \check{s} /$ and the vowel phonemes $/ i, I, e$, ae, $u, U, o, a /$ were used as stimuli. All consonant phonemes were paired with the vowel a/. The distinctive-feature composition of these phonemes, according to Halle's (1962, 1964) distinctive-feature system, is shown in Table 1 . It can be seen that each vowel phoneme is uniquely described by a + or - value on five acoustic dimensions, while each consonant phoneme is uniquely described by a binary value on six dimensions.

It can be seen in Table 1 that phonemes vary greatly in terms of their distinctive features. For example, /i/ and /e/ differ by only one distinctive feature, while /æ/ and /U/ differ by five distinctive features. Similarly $/ \mathrm{m} /$ and (b/ differ by a single distinctive feature, while $/ \mathrm{m} /$ and $/ \check{\mathrm{s}} /$ differ by six distinctive features.

To insure accurate reproduction of a particular stimulus on a given trial, the 16 consonant and vowel phonemes were recorded in a male voice and stored on disk tape in an IBM 360/44 computer. Each sound was cut to a length of exactly $250 \mathrm{msec}$ to insure simultaneity of onset and offset. The stimuli were recorded onto magnetic tape by attaching a Sony Model TC540 stereo tape recorder to the computer output and programming the computer to play the required phonemes simultaneously into separate channels of the tape recorder. The computer also recorded a 250-msec warning tone on Channel $A$ 2 sec prior to the onset of the stimuli. The onset of the stimulus recorded on
Table 1

Distinctive Feature Composition of Consonant and Vowel Phonemes Used in This Experiment

\begin{tabular}{|c|c|c|c|c|c|c|c|c|}
\hline & lb/ & $/ \mathrm{m} /$ & $|\mathrm{v}|$ & $|\mathrm{g}|$ & $/ \mathbf{d} /$ & $/ \mathbf{n} /$ & $|z|$ & $|s|$ \\
\hline Grave & + & + & + & + & - & - & - & - \\
\hline Diffuse & + & + & + & - & + & + & + & - \\
\hline Strident & - & - & + & - & - & - & + & + \\
\hline Nasal & - & + & - & - & - & + & - & - \\
\hline Continuant & - & - & + & - & - & - & + & + \\
\hline \multirow[t]{2}{*}{ Voiced } & + & + & + & + & + & + & + & - \\
\hline & iil & $/ \mathbf{I} /$ & $|\mathbf{e}|$ & $1 \infty 1$ & 101 & $10 I$ & $10 /$ & $|a|$ \\
\hline Flat & - & - & - & - & + & + & + & - \\
\hline Compact & - & - & - & + & - & - & - & + \\
\hline Diffuse & + & + & - & - & + & + & - & - \\
\hline Grave & - & - & - & - & + & + & + & + \\
\hline Tense & + & - & + & + & + & - & + & - \\
\hline
\end{tabular}




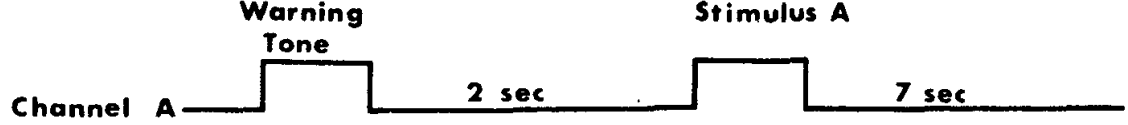

250 msec

250 msec

Stimulus B

Channel

\section{$250 \mathrm{msec}$}

Fig. 1. Placement of stimuli on a single dichotic trial.

Channel B activated a Hunter 100-msec timer, which was stopped by S's "same-different" response to the two stimuli. A 7-sec pause separated successive trials. A typical stimulus sequence is illustrated in Fig. 1.

Procedure. Ss were assigned randomly to either the consonant group or the vowel group. Each $\mathbf{S}$ was presented with 224 trials consisting of each sound paired with itself 14 times for a total of 112 "same" trials and paired with each of the other seven sounds twice (once to each ear) for a total of 112 "different" trials. "Same" and "different" trials were presented in random order.

Each $\mathbf{S}$ was seated at a small table, holding a two-way toggle switch grasped between his thumb and forefinger. $\mathrm{S}$ was instructed to move the switch to the right as quickly as possible when the stimuli in both ears were the same and to the left when the stimuli were different. Stimuli were presented to $S$ via a Sony Model TC540 stereo tape recorder and Koss Pro4A stereo headphones, so that stimuli were heard simultaneously in both ears. After each trial, E recorded S's RT. The experiment commenced following 10 practice trials.

Results

Analysis of variance revealed that RT for "same" responses was not significantly faster than RT for "different" responses. However, the faster RT for consonants than for vowels ( 595 vs $716 \mathrm{msec}$ ) was significant at the .05 level $(F=4.12$, $\mathrm{df}=1 / 38$ ). There was no interaction between phoneme type (consonant vs vowel) and response type ("same" vs "different").

Figure 2 describes the RT to pairs of vowel phonemes differing by 0 to 5 distinctive features and pairs of consonants differing by 0 to 6 distinctive features. For both consonants and vowels, there was an orderly decrease in RT to phonemes sharing fewer distinctive features.
Analysis of variance revealed that $\mathrm{RT}$ varied as a function of the distinctive-feature similarity of the stimulus pair $(F=7.07$, df $=5 / 190$, $\mathrm{p}<.001)$. Again, there was no interaction between phoneme type and phonemic distinctive-feature similarity.

One possible explanation of these data is that $S$ was responding "same" or "different" to each pair of sounds based on their apparent localization in his head. For example, "same" responses might be based on hearing the two sounds fuse into a single sound near the center of the head, while "different" responses would be based on hearing the sounds as further apart. Since distinctive features provide a general description of the physical similarity of any two sounds, pairs differing by many distinctive features could be localized furthest apart in the head.

If this reasoning is correct, one would expect to find an orderly relationship between the distinctive-feature similarity of two sounds and their subjective localization in the head. To test this hypothesis, 20 naive Ss were randomly presented with 58 pairs of either consonants or vowels, and asked to localize the two sounds. S localized the sound by placing the appropriate mark(s) on a semicircle representing the top 90 deg of a circle with a radius of $32 \mathrm{~mm}$.

Table 2 displays the mean separation in millimeters for pairs of consonants and vowels differing by 0 to 6 distinctive features. For both consonants and vowels, there was a marked tendency for Ss to respond to identical stimuli at each ear by placing a single mark at the center (top) of the semicircle. For consonants, there was a direct relationship between degree of separation and distinctive-feature similarity: the more different two sounds in terms of their distinctive features, the further apart they were localized. No such tendency was observed for vowels.
EXPERIMENT 2

The localization data revealed that $S$ could be responding "different" to pairs of consonants based on their subjective localization, rather than comparing the sounds in terms of their distinctive features (of course, it is possible that localization could be based on a distinctive-feature comparison). In order to eliminate S's ability to localize the consonant sounds, an experimental tape was prepared in which the two stimuli on each trial were always presented in different voices. Thus, one sound on each trial was spoken in a male voice, while the other was spoken in a female voice. This manipulation does not affect the relative distinctive-feature similarities of the sounds. However, the localization data from 10 Ss presented with 58 mixed-voice pairs revealed that $S$ always heard the two sounds at each ear, even when the same syllable was presented to both ears. Since presenting the stimuli in two voices eliminates localization effects due to the distinctive-feature similarity of the consonant phonemes, Ss were presented with these stimuli for same-different judgments in a RT experiment.

\section{Subjects}

Twenty right-handed undergraduate students from the University of Waterloo served as Ss. All Ss were students in an introductory psychology course, although participation was voluntary.

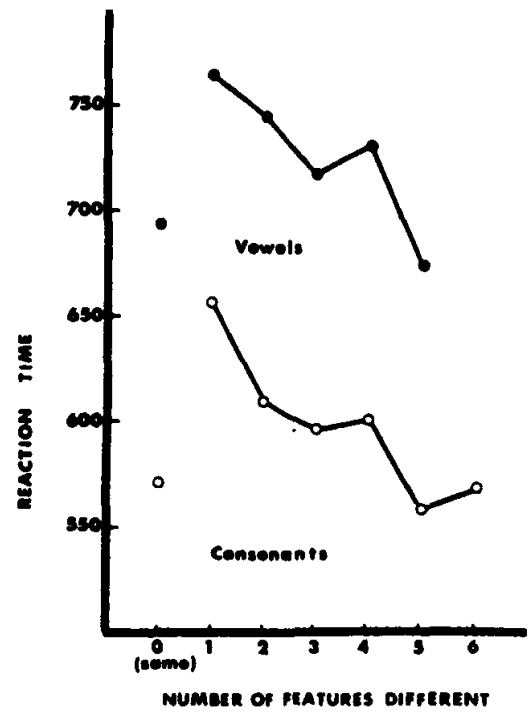

Fig. 2. Mean RT to dichotically presented consonants differing by 0 to 6 distinctive features and to vowels differing by 0 to 5 distinctive features. Data were summed over $20 \mathrm{Ss}$ in each condition. 
Stimuli

The consonant phonemes displayed in Table 1 were used as stimuli. Each consonant paired with /a/ was recorded in a male voice and a female voice, and stored on disk tape in an IBM 360/44 computer. A stimulus tape was then made, using the same pairs of stimuli presented in Experiment 1. However, on each trial, the syllable presented to one ear was heard in a male voice, while the syllable presented to the other ear was heard in a female voice. For each possible pairing of the stimuli, the male and female voices were heard equally often in the left and right ears.

\section{Procedure}

The experimental procedure was identical in Experiments 1 and 2.

\section{Results}

Figure 3 displays $\mathrm{RT}$ to pairs of consonants differing by 0 to 6 distinctive features. It is notable that RTs to all pairs were significantly longer in Experiment 2 than in Experiment 1. Analysis of variance revealed no differences in RT to "same" or "different" pairs. However, there were significant differences in $\mathrm{RT}$ to pairs differing by 1 to 6 distinctive features $(F=7.68, \mathrm{df}=$ $5 / 95, \quad p<.01)$. A trend analysis revealed a significant linear component ( $p<.02)$ to the decreasing function displayed in Fig. 3.

\section{DISCUSSION}

The present experiment demonstrates that same-different judgments about simultaneously heard phonemes are based on a comparison of their distinctive features. The direct relationship between $R T$ and distinctive-feature similarity for syllables differing by a single phoneme suggests a serial self-terminating model, as discussed by Bamber (1969). According to this model, two stimuli dimensions until a differentiating feature is found which terminates processing and elicits a "different" response. The more dimensions by which two stimuli differ, the faster a discriminating feature will be located, resulting in a faster $R T$.

If comparisons were based only on a serial self-terminating model, then "same" responses would take longer than the slowest "different" responses. are serially scanned along a number of

However, the data from this and other experiments (e.g., Egeth, 1966, Bamber, 1969) reveal that "same" responses are at least as fast as the fastest "different" responses. To account for this discrepancy in the fast RT for "same" responses, Bamber (1969) hypothesized an independent process, called the "identity reporter," which responds only to identical stimuli. Egeth and Blecker (1971) recently found that RT for "same" and "different" responses may be differentially influenced by the familiarity of the stimuli, a result which supports the independence of the two processes.

Several experiments have suggested that a "same" response may be based on a direct "physical" match of two stimuli. Evidence for a physical or sensory comparison has been demonstrated by Posner (1969) for visual stimuli and by Coltheart and Allard (1970) for auditory stimuli. While a physical match may occur in some experiments, it is clear that this process will not account for the fast RT of "same" responses for syllables heard in different voices. In this case, S must respond "same" without making a physical match.

The results of experiments which require recognition of dichotically presented phonemes rather than same-different judgments suggest that decisions concerning the names of two phonemes may involve a third level of processing. This contention is supported by an examination of the number and types of errors made in the two types of experiments. In the present experiment, there were very few errors (less than 2\%), and these were mostly anticipatory errors in which S knew immediately whether or not he had made an error. On the other hand, experiments requiring Ss to name the phonemes presented to each ear yield a large number of errors. For example, Ss in a dichotic listening experiment performed by Shankweiler and Studdert-Kennedy (1967) averaged only $40 \%$ accuracy in identifying the consonant or vowel presented to each ear. Thus, Ss are able to decide with great accuracy whether two simultaneously heard phonemes are the same or different, but have great difficulty in naming the individual phonemes. These results suggest that a same-different response and an identification response engage

Table 2

Mean Distance in Millimeters for Sounds Subjectively Localized in the Head

\begin{tabular}{llllllll}
\hline & 0 & 1 & 2 & 3 & 4 & 5 & 6 \\
\hline Vowels & .9 & 23 & 25 & 23 & 26 & 25 & 34 \\
Consonants & 1 & 26 & 28 & 29 & 30 & 33 & 34 \\
\hline
\end{tabular}

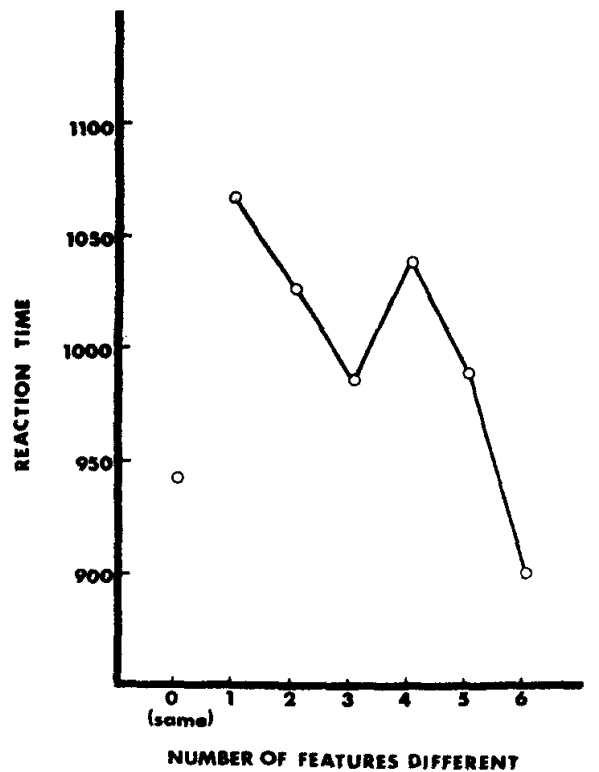

Fig. 3. Mean RT to dichotically presented consonants differing by 0 to 6 distinctive features. The two consonants presented on each trial were spoken in a different voice.

different processing levels. It is likely that Ss decide that two phonemes are "different" on the basis of their distinctive features before naming the individual phonemes presented to each ear.

It appears that recognition of simultaneously heard phonemes also requires identification of their distinctive features. Halwes (1969) required $S s$ to identify pairs of dichotically presented CVC syllables that differed by a single phoneme. In a very sophisticated analysis, Halwes discovered that recognition errors resulted from the "blending" of the distinctive features presented to each ear. For example, /b/ (front, voiced) and $/ t /$ (middle, unvoiced) combine to produce / $p$ / (front, unvoiced), which is a recombination of the individual features in $/ b /$ and $/ t /$. These and other studies (e.g., Miller \& Nicely, 1955) suggest that phonemes are recognized as sets of independently processed distinctive features.

In summary, it has been argued that two processes are involved in same-different judgments of simultaneously heard phonemes, and that a third process is involved in naming simultaneously heard phonemes. The present experiment suggests that "same" responses occur too fast to be accounted for by a serial self-terminating scan, suggesting that "same" and "different" responses involve independent processes. Deciding whether two phonemes are different seems to require that $\mathrm{S}$ 
compare phonemes in terms of their distinctive features. This comparison can be accomplished without regard to the ear in which the phoneme was heard. As long as $S$ is not required to recognize the phonemes, he has only to decide whether the feature values on any of several independently processed dimensions are different. In order to recognize the phonemes presented to each ear, $\mathbf{S}$ must retain laterality information for each of these features. The identification of the complete set of features presented to each ear thus requires a more complex analysis than that involved in a same-different judgment.

\section{REFERENCES}

BAMBER, D. Reaction times and error rates for "same" "different" judgments of multid:mensional stimuli. Perception \&
Psychophysics, 1969, 6, 169-174.

CHANANIE, J. D., \& TIKOFSKY, R. S Reaction time and distinctive features in speech discrimination. Paper presented at the meeting of the American Psychological Association, San Francisco. 1968.

COLTHEART, M., \& ALLARD, F Variations on a theme by Posner: Physical and name codes of heard letters. Paper presented at the 10th annual meeting of the Psychonomic Society, San Antonio, November 1970.

EGETH, H. E. Parallel versus serial processes in multidimensional stimulus discrimination. Perception Psychophysics, 1966, 1, 245-252.

EGETH, H., \& BLECKER, D. Differential effects of familiarity on judgment of sameness and difference. Perception \& Psychophysics, 1971, 9, 321-326.

HALLE, $M$. Phonology in generative grammar. Word, 1962, 18, 54-72.

HALLE, M. On the bases of phonology. In J. A. Fodor and J. J. Katz (Eds.), The structure of language. Englewood Cliffs. N.J: Prentice-Hall, 1964.
HALWES, T. G. Effects of dichotic fusion on the perception of speech. Supplement to: Status Report on Speech Research, MeINISH, J. R., \& TIKOFSKY, R. S Distinctive features and response latency: A pilot study. Perception \& Psychophysics, 1969,6, 267-268.

MILLER, G., \& NICELY, P. An anaiysis of perceptual confusions among some English consonants. Journal of the Acoustical Society of America, 1955, 27. 338-352.

POSNER. M. Abstraction and the process of recognition. In J. T. Spence and G. Bower (Eds.), The psychology of learning and motivation. Vol. 3. New York: Academic Press, 1969

S $H A$ A K W E I L E R, D . , \& STUDDERT-KENNEDY, $M$. An analysis of perceptual confusions in identification of dichotically presented CVC syllables. Quarterly Journal of Experimental Psychology, 1967, XIX, 59-63.

(Accepted for publication April 10,1972.) 